\author{
Nr. 151/93 \\ LaW of Large Numbers and Central Limit \\ THEOREM FOR DONSKER'S DELTA FUNCTION \\ OF DifFUSIONS I
}

J. Potthoff and P. Sundar 


\title{
Law of Large Numbers and Central Limit Theorem for Donsker's Delta Function of Diffusions I
}

\author{
J. Potthoff \\ Lehrstuhl für Mathematik V, Universität Mannheim, Mannheim, Germany, and \\ Department of Mathematics, Louisiana State University, Baton Rouge, USA \\ P. Sundar ${ }^{\ddagger *}$ \\ Department of Mathematics, Louisiana State University, Baton Rouge, USA
}

\begin{abstract}
Limit theorems in the space of Hida distributions, similar to the law of large numbers and the central limit theorem, are shown for composites of the Dirac distribution with solutions of one-dimensional, non-degenerate Itô equations.
\end{abstract}

$\dagger$ Supported by National Science Foundation under grant DMS - 9001859 .

† Supported by the Louisiana Education Quality Support Fund under grant (91-93) RD-A-08.

* Supported by the Council on Research of Louisiana State University. 


\section{Introduction}

In this paper we continue our work begun in [PS 92] on the construction of limits for the composition of the Dirac distribution $\delta_{x}$ concentrated at $x \in \mathbb{R}^{d}$ with an $\mathbb{R}^{d}$-valued process $X$ over $[0,1]$. In our first paper [PS 92] we established limit theorems resembling the law of large numbers (LLN) and the central limit theorem (CLT) in the case that $X$ is a one-dimensional Gaussian process. The limits were taken in the sense of strong limits of Hida distributions. (In case of the LLN we also proved weak convergence in the Meyer-Watanabe space.) In the present paper we extend these results to solutions of one-dimensional, non-degenerate Itô equations. Our final aim is to show such limit results for systems of interacting diffusions, and constructing solutions of the McKean-Vlasov equation.

LLN and CLT for generalized random variables have also been considered independently in [Am 92]. The relation between the results there and in the present paper has still to be worked out.

As in our first paper [PS 92] we work in the framework of white noise calculus. Our proofs use a combination of results from white noise analysis (in particular from [PS 91]) and of techniques from Malliavin calculus. The methods of the present paper work as well in the general case of $d$-dimensional diffusion processes, that arise as solutions of nondegenerate Ito equations. However, for $d>2$ the calculation of the $\mathcal{S}$-transform given in Section 3 for $d=1$ has to incorporate higher order derivatives, and therefore necessitates the estimation of much more complex terms in the resulting Malliavin calculus. Also, as a result formula (5.2) for the "variance" appearing in the central limit theorem is more complicated for arbitrary $d \in \mathbb{N}$. Since the additional complications which come up by treating the case of general $d$ would obfuscate thoroughly the arguments of our proofs, we consider in the present paper only the one-dimensional case, and in a separate forthcoming paper the situation of arbitrary $d$.

The paper is organized as follows. In Section 2 we recall quickly some facts from white noise and Malliavin calculus. In Section 3 we prove the essential technical result: we derive a formula for the $\mathcal{S}$-transform of $\delta_{x} \circ X$ for non-degenerate $X$ belonging to the Meyer-Watanabe space $\mathcal{D}$. In Section 4 we prove the LLN, in Section 5 the CLT. In an Appendix we collect some technical results related to isometric transformations of the underlying probability space.

\section{Some Background and Results From Stochastic Calculus}

In this section we give a brief sketch of pertinent notions and results from stochastic calculus. For more details we refer (for example) to [HK 92], and the literature quoted there. As in our previous paper [PS 92], we continue to use white noise analysis as our general framework, and the interested reader may consult Section 2 of [PS 92] for a quick introduction.

Let $\left(\mathcal{S}^{\prime}(\mathbb{R}), \mathcal{B}, \mu\right)$ be the probability space of white noise. I.e., $\mu$ is the centered Gaussian measure on the weak Borel $\sigma$-algebra $\mathcal{B}$ of $\mathcal{S}^{\prime}(\mathbb{R})$ whose covariance is given by 
the inner product of $L^{2}(\mathbb{R}, d t) \equiv L^{2}(\mathbb{R}), d t$ denoting Lebesgue measure. In Sections 4 and 5 we shall prove limit theorems in the space $(\mathcal{S})^{*}$ of Hida distributions which is the dual of a certain space $(\mathcal{S})$ of smooth functions on $\mathcal{S}^{\prime}(\mathbb{R})$. For a construction of $(\mathcal{S})$, and for properties of $(\mathcal{S})$ and $(\mathcal{S})^{*}$ we refer to [HK 92, PS 91]. The canonical dual pairing between $(\mathcal{S})^{*}$ and $(\mathcal{S})$ is denoted by $\langle\cdot, \cdot>$.

In the proofs of the following sections, an essential role is played by the infinite dimensional gradient $\nabla$, and the associated directional derivatives. Natural domains for these differential operators (in the context of the present paper) are the spaces $\mathcal{D}^{p, k}, p>1, k \in \mathbb{Z}$, introduced by Meyer [Me 83] (cf. also, e.g., [Su 85, Wa 83, Wa 84]). Roughly speaking we have

$$
\mathcal{D}^{p, k}:=(1+N)^{-k / 2} L^{p}(\mu)
$$

where $N$ is the number operator (i.e., the negative of the Ornstein-Uhlenbeck operator). The norm of $\mathcal{D}^{p, k}$ will be denoted by $\|\cdot\|_{p, k}$. Let $h \in L^{2}(\mathbb{R})$, and consider the Gâteaux derivative $D_{h}$ in direction $h$ acting on an appropriate function $\varphi$ on $\mathcal{S}^{\prime}(\mathbb{R})$ :

$$
\left(D_{h} \varphi\right)(\omega)=\left.\frac{d}{d \lambda} \varphi(\omega+\lambda h)\right|_{\lambda=0}
$$

whenever the right hand side exists. The Meyer inequalities (e.g., [Me 83, Su 85]) give the estimate

$$
\left\|D_{h} \varphi\right\|_{p, k} \leq C_{p, k}|h|_{2}\|\varphi\|_{p, k+1},
$$

where $|\cdot|_{2}$ is the norm of $L^{2}(\mathbb{R})$ and $C_{p, k}>0$ is a constant. Clearly, this entails that $D_{h}$ extends from a suitable dense class of functions (say, smooth cylinder polynomials) to a continuous operator from $\mathcal{D}^{p, k}$ into $\mathcal{D}^{p, k-1}$. Moreover, if $\varphi \in \mathcal{D}^{p, k}, k \geq 1$, then the mapping $h \longmapsto\left(D_{h} \varphi\right)(\omega)$ defines for $\mu$-a.e. $\omega \in \mathcal{S}^{\prime}(\mathbb{R})$ a continuous linear mapping from $L^{2}(\mathbb{R})$ into $\mathbb{C}$. Therefore there exists an element $\nabla \varphi \in L^{2}(\mathbb{R}) \otimes \mathcal{D}^{p, k-1}$, such that for every $h \in L^{2}(\mathbb{R})$ and $\mu$-a.e. $\omega \in \mathcal{S}^{\prime}(\mathbb{R})$ we have

$$
D_{h} \varphi(\omega)=(h, \nabla \varphi(\omega)),
$$

where the inner product on the right hand side is the one of $L^{2}(\mathbb{R})$. Also, it is well-known that the adjoint $D_{h}^{*}$ of $D_{h}$ is given by

$$
D_{h}^{*} \varphi(\omega)=<\omega, h>\varphi(\omega)-D_{h} \varphi(\omega),
$$

(where equality is in the sense of $L^{2}(\mu)$ ) and that $N=\nabla^{*} \nabla$, where $\nabla^{*}$ is the adjoint of $\nabla($ with respect to $d t \otimes d \mu)$.

In order to apply the results of the following sections to diffusion processes, we will apply the important result of Malliavin calculus which states that the solutions of nondegenerate Itô equations belong to the Meyer-Watanabe space $\mathcal{D}$, which is the intersection of the spaces $\mathcal{D}^{p, k}$, and that these solutions have a non-degenerate Malliavin covariance matrix. The dual of the space $\mathcal{D}(\mathcal{D}$ being equipped with the projective limit topology) is denoted by $\mathcal{D}^{*}$, and the dual pairing between these spaces is also denoted by $\langle\cdot, \cdot\rangle$. (There is no danger of confusion because $(\mathcal{S})$ is continuously embedded into $\mathcal{D}$.) 
A crucial role in the proofs of the LLN and the CLT is played the $\mathcal{S}$-transform

$$
\mathcal{S} \Phi(\xi)=\left\langle\bar{\Phi},: e^{<\cdot \xi\rangle}:>, \quad \xi \in \mathcal{S}(\mathbb{R}),\right.
$$

defined for all $\Phi \in(\mathcal{S})^{*}$, because the function

$$
\omega \longmapsto: e^{<\omega, \xi>}: \equiv e^{<\omega, \xi>-\frac{1}{2}|\xi|_{2}^{2}},
$$

belongs to $(\mathcal{S})$. Pointwise convergence of the $\mathcal{S}$-transforms of a sequence in $(\mathcal{S})^{*}$, together with a certain uniform bound, is equivalent to strong convergence in $(\mathcal{S})^{*}$ [PS 91].

\section{Calculating the $\mathcal{S}$-Transform of $\delta_{x} \circ X$}

This section contains the technical key result which will be used in Sections 4 and 5 to derive the LLN and the CLT for certain versions of $\delta_{x} \circ X$.

Throughout this paper we shall assume that $X$ is a real random variable in $\mathcal{D}$, that the mapping

$$
\omega \longmapsto\left(|\nabla X|_{2}(\omega)\right)^{-1}
$$

exists $\mu$-a.e., and that it belongs to every $L^{p}(\mu), p \geq 1$.

Theorem 3.1. Let

$$
F(X):=N X+2|\nabla X|_{2}^{-2}\left(\nabla^{2} X,(\nabla X)^{\otimes 2}\right)_{L^{2}\left(\mathbb{R}^{2}\right)}
$$

For all $x \in \mathbb{R}, \xi \in \mathcal{S}(\mathbb{R})$,

$$
\left(\mathcal{S} \delta_{x} \circ X\right)(\xi)=\int_{\{X \geq x\}}|\nabla X|_{2}^{-2}\left(F(X)-D_{\xi} X\right): e^{<\cdot \xi>}: d \mu .
$$

In passing we mention the following consequence of Theorem 3.1 which might be of independent interest:

Corollary 3.2 Assume that $f \in C(\mathbb{R})$ is polynomially bounded. Then

$$
(\mathcal{S} f \circ X)(\xi)=\int f(x) \int_{\{X \geq x\}}|\nabla X|_{2}^{-2}\left(F(X)-D_{\xi} X\right): e^{<\cdot \xi\rangle}: d \mu d x
$$

Theorem 3.1 is proved by the next two lemmas. 
Lemma 3.3. For all $x \in \mathbb{R}, \varphi \in \mathcal{D}$,

$$
<\delta_{x} \circ X, \varphi>=\int_{\{X \geq x\}} \nabla^{*}\left((\nabla X)|\nabla X|_{2}^{-2} \varphi\right) d \mu .
$$

Proof. By definition, $\delta_{x} \circ X$ is the unique element in $\mathcal{D}^{*}$ which is obtained as the strong limit of $\delta_{x, n} \circ X$, where $\left\{\delta_{x, n}, n \in \mathbb{N}\right\}$ is any sequence in $\mathcal{S}^{\prime}(\mathbb{R})$ converging strongly to $\delta_{x}$ as $n$ tends to infinity (cf. [Wa 83]).

For $n \in \mathbb{N}$, let $\theta_{x, n}$ be any monotonically increasing $C^{\infty}$-function, so that $\theta_{x, n}=0$ on $\left(-\infty, x-\frac{1}{n}\right]$, and $\theta_{x, n}=1$ on $[x,+\infty)$. Then $\theta_{x, n}^{\prime}$ converges strongly in $\mathcal{S}^{\prime}(\mathbb{R})$ to $\delta_{x}$. Following Malliavin [Ma 78], we use the chain rule to write

$$
\left(\nabla X, \nabla \theta_{x, n} \circ X\right)_{L^{2}(\mathbb{R})}=\theta_{x, n}^{\prime} \circ X \cdot|\nabla X|_{2}^{2} .
$$

Thus we have

$$
\begin{aligned}
<\theta_{x, n}^{\prime} \circ X, \varphi> & =<\left(\nabla X, \nabla \theta_{x, n} \circ X\right),|\nabla X|_{2}^{-2} \varphi> \\
& =\int \theta_{x, n} \circ X \nabla^{*}\left((\nabla X)|\nabla X|_{2}^{-2} \varphi\right) d \mu .
\end{aligned}
$$

Our hypotheses on $X$ entail that all quantities under the integral are well defined. Letting $n$ tend to infinity, by Watanabe's result [Wa 83$]$ the left hand side converges to $\left\langle\delta_{x} \circ X, \varphi\right\rangle$. On the other hand the dominated convergence theorem applied to the integral expression shows that this term converges to the right hand side of (3.2).

Lemma 3.4. For all $x \in \mathbb{R}, \varphi \in \mathcal{D}$,

$$
<\delta_{x} \circ X, \varphi>=\int_{\{X \geq x\}}|\nabla X|_{2}^{-2}(F(X) \varphi-(\nabla X, \nabla \varphi)) d \mu .
$$

Proof. The product rule (for suitable $\varphi, \psi$ ) of $D_{h}^{*}$ reads

$$
D_{h}^{*} \varphi \psi=\left(D_{h}^{*} \varphi\right) \psi-\varphi D_{h} \psi
$$

Moreover, we note that $D_{h}^{*}=\left(h, \nabla^{*}\right)$. Working out equation (3.2), and using $N=\nabla^{*} \nabla$, we get immediately (3.3).

Theorem 3.1. follows now from the choice $\varphi=: e^{<\cdot, \xi\rangle}:, \xi \in \mathcal{S}(\mathbb{R})$, in (3.3).

In order to prepare the computations of the next sections we prove now a modification of Theorem 3.1. Assume that $\tau$ is a linear unitary mapping on $L^{2}(\mathbb{R})$, whose restriction to $\mathcal{S}(\mathbb{R})$ is a homeomorphism of $\mathcal{S}(\mathbb{R})$. Then $\tau$ and $\tau^{*}=\tau^{-1}$ extend to $\mathcal{S}^{\prime}(\mathbb{R})$. Let us define

$$
X_{\tau}(\omega):=X(\tau \omega), \quad \omega \in \mathcal{S}^{\prime}(\mathbb{R}) .
$$

It is clear that $\mu$ is invariant under $\tau: \mu \circ \tau=\mu$. Moreover, we show in the Appendix that all quantities in (3.1) not involving $\xi$ transform "covariantly" under $\tau$, i.e.. we have the following relations (cf. Lemma A.1 and Lemma A.2):

(i) $F\left(X_{\tau}\right)=(F(X))_{\tau}$,

(ii) $\left|\nabla X_{\tau}\right|_{2}=\left(|\nabla X|_{2}\right)_{\tau}$,

(iii) $D_{h} X_{\tau}=\left(D_{\tau h} X\right)_{\tau}$.

Thus, we arrive at the following result. 
Theorem 3.5. For all $x \in \mathbb{R}, \xi \in \mathcal{S}(\mathbb{R})$,

$$
\left(\mathcal{S} \delta_{x} \circ X_{\tau}\right)(\xi)=\int_{\{X \geq x\}}|\nabla X|_{2}^{-2}\left(F(X)-D_{\tau \xi} X\right): e^{<\cdot, \tau \xi>}: d \mu .
$$

\section{Law of Large Numbers}

In this section we prove a limit theorem for $\delta_{x} \circ X$ which resembles the law of large numbers. In particular, $X$ may be chosen as the solution of a non-degenerate Itô equation evaluated at $t>0$.

For simplicity, we shall consider processes over the unit time interval $[0,1]$. Let $B(t)$ denote the version of Brownian motion given by $\omega \longmapsto<\omega, 1_{[0, t]}>, t \in[0,1]$. $\mathcal{B}_{t}$ denotes the sub- $\sigma$-algebra of $\mathcal{B}$ generated by $\{B(s), s \in[0, t]\}$. (In other words, $\mathcal{B}_{t}$ is generated by the random variables $\omega \longmapsto<\omega, \xi>$, with $\xi \in \mathcal{S}(\mathbb{R})$, and $\operatorname{supp} \xi \subset[0, t]$.). From now on let $t>0$, and consider a real random variable $X$ satisfying the following hypotheses:

(H.1) $X$ is $\mathcal{B}_{t}$-measurable;

(H.2) $X \in \mathcal{D}$;

(H.3) $X$ has a non-degenerate Malliavin variance, i.e., $|\nabla X|_{2}$ is $\mu$-a.e. non-zero, and $|\nabla X|_{2}^{-1}$ belongs to all $L^{p}(\mu), p \geq 1$.

Similar to [PS 92], we construct independent versions of $X$ on $\left(\mathcal{S}^{\prime}(\mathbb{R}), \mathcal{B}, \mu\right)$ by means of a family of unitary transformations on $L^{2}(\mathbb{R})$. As in the case of Gaussian random variables considered in [PS 92], the transformations $\tau_{n}$ given by

$$
\left(\tau_{n} f\right)(s)=f(s+n), \quad n \in \mathbb{N},
$$

can be used to prove a LLN for $\delta_{x} \circ X_{n}$, where $X_{n}(\omega)=X\left(\tau_{n} \omega\right)$. However, as pointed out in [PS 92], these versions of $X$ are not suitable to prove a CLT. Thus - as in our previous paper - we use the following transformations:

$$
\left(\tau_{n, m} f\right)(s):=\frac{1}{\sqrt{n}} f\left(\frac{m+s}{n}\right), \quad n \in \mathbb{N}, m=0,1, \ldots, n-1 .
$$

(It is obvious that $\tau_{n, m}$ satisfies the conditions stated before Theorem 3.5.) For a random variable $Y$, define $Y_{n, m}:=Y\left(\tau_{n, m} \omega\right), \omega \in \mathcal{S}^{\prime}(\mathbb{R}), n \in \mathbb{N}, m=0,1, \ldots, n-1$. Since $\mu$ is invariant under $\tau_{n, m}$, we have for all $\lambda \in \mathbb{R}, n \in \mathbb{N}, m=0,1, \ldots, n-1$,

$$
\int \exp \left(i \lambda X_{n, m}\right) d \mu=\int \exp (i \lambda X) d \mu
$$

showing that $X_{n, m}$ and $X$ have the same distribution for all $n, m$. Moreover, $X_{n, m}$ is measurable with respect to the sub- $\sigma$-algebra $\mathcal{B}_{n, m, t}$ generated by $\left\{B_{n, m}(s), s \in[0, t]\right\}$. Since $B_{n, m}(s)(\omega)=\sqrt{n}<\omega, 1_{\left[\frac{m}{n}, \frac{m+e}{n}\right]}>$, it is clear that for every $n \in \mathbb{N}$, the Brownian motions $B_{n, m}$ and $B_{n, m^{\prime}}, m \neq m^{\prime}, \stackrel{n}{m}, m^{\prime}=0,1, \ldots, n-1$, on $[0,1]$ are independent. Thus $\mathcal{B}_{n, m, t}$ and $\mathcal{B}_{n, m^{\prime}, t}$ are independent for all $t \in[0,1]$. Consequently, $X_{n, m}$ and $X_{n, m^{\prime}}$ are independent. Altogether we have proved 
Proposition 4.1. For each $n \in \mathbb{N}$, the family

$$
\left\{X_{n, m}, m=0,1, \ldots, n-1\right\}
$$

is a family of i.i.d. versions of $X$.

For the proof of the LLN and the CLT the following (almost obvious) result will be useful.

Lemma 4.2. For every $h \in L^{2}(\mathbb{R})$,

$$
D_{h} X=D_{P_{t} h} X
$$

where $P_{t}$ is the orthogonal projection in $L^{2}(\mathbb{R})$ onto $L^{2}([0, t])$ (i.e., $P_{t}$ is multiplication by $\left.1_{[0, t]}\right)$.

Proof. Let $\left\{f^{(n)}, n \in \mathbb{N}_{0}\right\}$ be the sequence in the symmetric Fock space over $L^{2}(\mathbb{R})$ which gives the chaos decomposition of $X$ :

$$
X=\sum_{n=0}^{\infty} I_{n}\left(f^{(n)}\right)
$$

where $I_{n}\left(f^{(n)}\right)$ denotes the $n$-fold multiple Wiener integral with respect to the Brownian motion $B$. Then the measurability hypothesis (H.1) entails that $f^{(n)} \in L^{2}\left(\mathbb{R}^{n}\right)(\cdot$ denotes symmetrization) is of the form $f^{(n)}=g^{(n)} \cdot 1_{[0, t]^{n}}$, for some $g^{(n)} \in L_{\text {loc }}^{2}\left(\mathbb{R}^{n}\right)$. Since

$$
D_{h} X=\sum_{n=1}^{\infty} n I_{n-1}\left(\left(f^{(n)}, h\right)\right)
$$

where

$$
\left(f^{(n)}, h\right)\left(s_{1}, \ldots, s_{n-1}\right)=\int f^{(n)}\left(s_{1}, \ldots, s_{n-1}, s_{n}\right) h\left(s_{n}\right) d s_{n},
$$

the statement of the lemma is now obvious.

Now we are ready for the proof of the LLN.

Theorem 4.3. As $n$ tends to infinity,

$$
\frac{1}{n} \sum_{m=0}^{n-1} \delta_{x} \circ X_{n, m}
$$

converges strongly in $(\mathcal{S})^{*}$ to $\mathbb{E}\left(\delta_{x} \circ X\right):=<\delta_{x} \circ X, 1>$. Moreover,

$$
\mathbb{E}\left(\delta_{x} \circ X\right)=\int_{\{X \geq x\}}|\nabla X|_{2}^{-2} F(X) d \mu
$$


where

$$
F(X):=N X+2|\nabla X|_{2}^{-2}\left(\nabla^{2} X,(\nabla X)^{\otimes 2}\right)_{L^{2}\left(\mathbb{R}^{2}\right)}
$$

Proof. Observe that by hypotheses (H.2) and (H.3), $F(X) \in L^{p}(\mu)$, for all $p \geq 1$. By Theorem 3.5, the $\mathcal{S}$-transform of

$$
\frac{1}{n} \sum_{m=0}^{n-1} \delta_{x} \circ X_{n, m}
$$

at $\xi \in \mathcal{S}(\mathbb{R})$ is given by

$$
\frac{1}{n} \sum_{m=0}^{n-1} \int_{\{X \geq x\}}|\nabla X|_{2}^{-2}\left(F(X)-D_{\tau_{n, m} \xi} X\right): e^{<\cdot, \tau_{n, m} \xi>}: d \mu
$$

Let us first show that the term under the sum involving the derivative in direction of $\tau_{n, m} \xi$ vanishes. To this end, it suffices to prove that $D_{\tau_{n, m} \xi} X$ converges to zero in $L^{2}(\mu)$, uniformly in $m$, because obviously $|\nabla X|_{2}^{-2}: \exp <\cdot, \tau_{n, m} \xi>$ : belongs to $L^{2}(\mu)$, too, and is uniformly bounded in $n, m$ in $L^{2}(\mu)$. (Use Hölder's inequality, the fact that every term is in every $L^{p}(\mu), p \geq 1$, and $\left\|: \exp <\cdot, \tau_{n, m} \xi>:\right\|_{p}=\exp \left(\frac{1}{2}(p-1)|\xi|_{2}^{2}\right)$.) From Lemma 4.2 we know that

$$
D_{\tau_{n, m} \xi} X=D_{P_{\mathrm{t}} \tau_{n, m} \xi} X
$$

Now,

$$
P_{t} \tau_{n, m} \xi(s)=n^{-1 / 2} \xi\left(\frac{m+s}{n}\right) 1_{[0, t]}(s), \quad s \in \mathbb{R} .
$$

Thus we can estimate

$$
\begin{aligned}
\left\|D_{\tau_{n, m} \xi} X\right\|_{2} & =\left\|D_{P_{\mathrm{t}} \tau_{n, m} \xi} X\right\|_{2} \\
& \leq\left|P_{t} \tau_{n, m} \xi\right|_{2}\left\|N^{\frac{1}{2}} X\right\|_{2} \\
& \leq t^{1 / 2} n^{-1 / 2}|\xi|_{\infty}\|X\|_{2,1} .
\end{aligned}
$$

(The first inequality above is well-known (e.g., [HK 92]). For example, it follows from (4.1) and Schwarz' inequality, and it is a special case of the Meyer inequalities.) Clearly, this estimate shows that $D_{\tau_{n, m} \xi} X$ converges in $L^{2}(\mu)$ to zero, uniformly in $m$, as $n$ tends to infinity.

Next we show that as $n$ tends to infinity,

$$
\int_{\{X \geq x\}}|\nabla X|_{2}^{-2} F(X): e^{<\cdot, \tau_{n, m} \xi>}: d \mu
$$

converges to

$$
\int_{\{X \geq x\}}|\nabla X|_{2}^{-2} F(X) d \mu
$$


uniformly in $m=0,1 \ldots, n-1$. By assumption, $\theta_{x}(X)|\nabla X|_{2}^{-2} F(X)$ is $\mathcal{B}_{t}$-measurable. Decompose $\tau_{n, m} \xi=P_{t} \tau_{n, m} \xi+P_{t}^{\perp} \tau_{n, m} \xi$, where $P_{t}^{\perp}=\mathrm{id}-P_{t}$. Then $\omega \longmapsto<\omega, P_{t}^{\perp} \tau_{n, m} \xi>$ is independent of $\mathcal{B}_{t}$. Using the fact that $\int: \exp \langle\cdot, h\rangle: d \mu=1$, for all $h \in L^{2}(\mathbb{R})$, we can compute as follows:

$$
\begin{aligned}
\int_{\{X \geq x\}}|\nabla X|_{2}^{-2} F(X) & : e^{<\cdot, \tau_{n, m} \xi>}: d \mu \\
= & \int_{\{X \geq x\}}|\nabla X|_{2}^{-2} F(X): e^{<\cdot, P_{t} \tau_{n, m} \xi>}: d \mu \\
= & \exp \left(-\frac{1}{2 n} \int_{0}^{t} \xi\left(\frac{m+s}{n}\right)^{2} d s\right) \times \\
& \quad \times \int_{\{X \geq x\}}|\nabla X|_{2}^{-2} F(X) \exp \left(<\cdot, P_{t} \tau_{n, m} \xi>\right) d \mu
\end{aligned}
$$

In order to show that the last expression converges to $\int_{X>_{x}}|\nabla X|_{2}^{-2} F(X) d \mu(x)$ as $n$ tends to infinity, uniformly in $m=0,1, \ldots, n-1$, it is sufficient to prove the following two claims:

(i) $\exp \left(-\frac{1}{2 n} \int_{0}^{t} \xi\left(\frac{m+s}{n}\right)^{2} d s\right) \longrightarrow 1$, as $n \longrightarrow+\infty$, uniformly in $m$,

(ii) $\exp \left(<\cdot, P_{t} \tau_{n, m} \xi>\right) \longrightarrow 1$ in $L^{2}(\mu)$, as $n \longrightarrow+\infty$, uniformly in $m$,

because $1_{\{X \geq x\}}|\nabla X|_{2}^{-2} F(X)$ belongs to $L^{2}(\mu)$. Claim (i) is obviously true, since $\xi$ is bounded. Concerning (ii) we remark that $P_{t} \tau_{n, m} \xi$ converges in $L^{2}(\mathbb{R})$ to zero as $n$ tends to infinity, uniformly in $m$ :

$$
\begin{aligned}
\left|P_{t} \tau_{n, m} \xi\right|_{2}^{2} & =n^{-1} \int_{0}^{t} \xi\left(\frac{m+s}{n}\right)^{2} d s \\
& \leq t n^{-1}|\xi|_{\infty}^{2}
\end{aligned}
$$

Now we have

$$
\left\|e^{<\cdot, P_{t} \tau_{n, m} \xi>}-1\right\|_{2}^{2}=e^{2\left|P_{t} \tau_{n, m} \xi\right|_{2}^{2}}-2 e^{1 / 2\left|P_{\mathrm{t}} \tau_{n, m} \xi\right|_{2}^{2}}+1
$$

and therefore also the convergence claimed in (ii) above holds true.

Altogether we have proved so far that the $\mathcal{S}$-transform of

$$
\frac{1}{n} \sum_{m=0}^{n-1} \delta_{x} \circ X_{n, m}
$$

converges pointwise to $\mathcal{S} \delta_{x} \circ X(0)$, which is equal to $\mathbb{E}\left(\delta_{x} \circ X\right)$. By Theorem 2.7 in [PS 91] it now suffices to show that there exists constants $K_{1}, K_{2}>0$ so that for all $z \in \mathbb{C}, \xi \in \mathcal{S}(\mathbb{R}), n \in \mathbb{N}, m=0,1, \ldots, n-1$, we have

$$
\left|\left(\mathcal{S} \delta_{x} \circ X_{n, m}\right)(z \xi)\right| \leq K_{1} e^{K_{2}|z|^{2}|\xi|_{2}^{2}}
$$

in order to conclude the proof. 
$\left(\mathcal{S} \delta_{x} \circ X_{n, m}\right)(z \xi)$ is defined by analytic continuation, and is given as follows:

$$
\left(\mathcal{S} \delta_{x} \circ X_{n, m}\right)(z \xi)=\int_{\{X \geq x\}}|\nabla X|_{2}^{-2}\left(F(X)-z D_{\tau_{n, m} \xi} X\right): e^{\left.z<\cdot, \tau_{n, m} \xi\right\rangle}: d \mu
$$

where we used linearity of $h \longmapsto D_{h}$, and $: e^{z<\cdot, h>}:, h \in L^{2}(\mathbb{R})$, denotes the function

$$
\omega \longmapsto e^{z<\omega, h>-\frac{1}{2} z^{2}|h|_{2}^{2}} \text {. }
$$

By the triangular and Hölder's inequalities - we get

$$
\begin{aligned}
\left|\left(\mathcal{S} \delta_{x} \circ X_{n, m}\right)(z \xi)\right| \leq\left\||\nabla X|_{2}^{-2} F(X)\right\|_{2}\left\|: e^{\left.z<\cdot, \tau_{n, m} \xi\right\rangle}:\right\|_{2}+ \\
\quad+|z|\left\|\left.\nabla X\right|_{2} ^{-2}\right\|_{3}\left\|D_{\tau_{n, m} \xi} X\right\|_{3}\left\|: e^{z<\cdot, \tau_{n, m} \xi>}:\right\|_{3} .
\end{aligned}
$$

Now use

$$
\left\|: e^{z<\cdot h>}:\right\|_{p} \leq \exp \left(\frac{1}{2}|z|^{2} p|h|_{2}^{2}\right)
$$

and

$$
\begin{aligned}
\left\|D_{\tau_{n, m} \xi} X\right\|_{p} & \leq C_{p}\left|\tau_{n, m} \xi\right|_{2}\|X\|_{p, 1} \\
& =C_{p}|\xi|_{2}\|X\|_{p, 1},
\end{aligned}
$$

(cf. Section 2) to obtain a bound of the form

$$
\begin{aligned}
\left|\mathcal{S} \delta_{x} \circ X_{n, m}(z \xi)\right| & \leq K_{1}\left(1+|z||\xi|_{2}\right) e^{3 / 2|z|^{2}|\xi|_{2}^{2}} \\
& \leq \sqrt{e} K_{1} e^{2|z|^{2}|\xi|_{2}^{2}}
\end{aligned}
$$

where $K_{1}$ depends only on $X$.

\section{Central Limit Theorem}

We continue to consider a random variable $X$ satisfying hypotheses (H.1), (H.2), and (H.3) of Section 4, and the family $\left\{X_{n, m}, n \in \mathbb{N}, m=0,1, \ldots, n-1\right\}$ of independent copies of $X$. As in the previous sections, we denote

$$
F(X):=N X+2|\nabla X|_{2}^{-2}\left(\nabla^{2} X,(\nabla X)^{\otimes 2}\right)_{L^{2}\left(\mathbb{R}^{2}\right)} .
$$

Theorem 5.1. Let $X$ be a random variable satisfying conditions (H.1), (H.2), and (H.3). Then

$$
\frac{1}{\sqrt{n}} \sum_{m=0}^{n-1}\left(\delta_{x} \circ X_{n, m}-\mathbb{E}\left(\delta_{x} \circ X\right)\right)
$$

converges strongly in $(\mathcal{S})^{*}$ to $\sigma(t, x) Z$, as $n$ tends to infinity, where $Z$ is a standard normal random variable, and $\sigma(t, x)$ is given by

$$
\sigma(t, x)=\int_{\{X \geq x\}}|\nabla X|_{2}^{-2}\left(F(X) B(t)-D_{1_{[0, t]}} X\right) d \mu
$$


For the proof of Theorem 5.1 we prepare first the following simple result.

Lemma 5.2. Let $\xi \in \mathcal{S}(\mathbb{R}), \tau_{n, m}, n \in \mathbb{N}, m=0,1,2, \ldots, n-1$, and $P_{t}, t>0$, be as above. Then $n^{-1 / 2} \sum_{m=0}^{n-1} P_{t} \tau_{n, m} \xi$ converges in $L^{2}(\mathbb{R})$ to $\left(\int_{0}^{1} \xi(u) d u\right) \cdot 1_{[0, t]}$.

Proof. Let $s \in \mathbb{R}$, then we have by Taylor's theorem

$$
\left(n^{-1 / 2} \sum_{m=0}^{n-1} P_{t} \tau_{n, m} \xi\right)(s)=\left(\sum_{m=0}^{n-1} n^{-1} \xi\left(\frac{m}{n}\right)\right) 1_{[0, t]}(s)+O_{\xi, t}\left(n^{-1}\right) 1_{[0, t]}(s)
$$

where $\left|O_{\xi, t}\left(n^{-1}\right)\right| \leq t n^{-1}\left|\xi^{\prime}\right|_{\infty}$. Since $\xi$ is continuous, it is clear that the claimed convergence holds pointwise for every $s \in \mathbb{R}$. The statement of the lemma follows then by a simple application of the dominated convergence theorem.

Proof of Theorem 5.1. The $\mathcal{S}$-transform of (5.1) evaluated at $\xi \in \mathcal{S}(\mathbb{R})$ is given by (cf. Theorem 3.5)

$$
\begin{aligned}
& \frac{1}{\sqrt{n}} \sum_{m=0}^{n-1} \int_{\{X \geq x\}}|\nabla X|_{2}^{-2}\left(\left(F(X)-D_{\tau_{n, m} \xi} X\right): e^{<\cdot, \tau_{n}, m \xi>}:-F(X)\right) d \mu= \\
&=\frac{1}{\sqrt{n}} \sum_{m=0}^{n-1} \int_{\{X \geq x\}}|\nabla X|_{2}^{-2} F(X)\left(: e^{<\cdot, P_{\mathrm{t}} \tau_{n, m} \xi>}:-1\right) d \mu- \\
&-\frac{1}{\sqrt{n}} \sum_{m=0}^{n-1} \int_{\{X \geq x\}}|\nabla X|_{2}^{-2}\left(D_{P_{\mathrm{t}} \tau_{n, m} \xi} X\right): e^{<\cdot, P_{\mathrm{t}} \tau_{n, m} \xi>}: d \mu,
\end{aligned}
$$

where we used the same arguments as in the proof of Theorem 4.3 to reduce $\tau_{n, m} \xi$ to $P_{t} \tau_{n, m} \xi$. Consider the first term on the right hand side of (5.3). First we show that

$$
n^{-1 / 2} \sum_{m=0}^{n-1}\left(: e^{\left\langle\cdot, P_{t} \tau_{n, m} \xi\right\rangle}:-1\right)
$$

converges in $L^{2}(\mu)$ to $B(t) \int_{0}^{1} \xi(u) d u$, as $n$ tends to infinity. We do this in three steps:

(i) We prove that the $L^{2}(\mu)$-limit of $(5.4)$ is equal to the $L^{2}(\mu)$-limit of

$$
n^{-1 / 2} \sum_{m=0}^{n-1}\left(e^{<\cdot, P_{t} \tau_{n, m} \xi>}-1\right) \text {. }
$$

Indeed, using the triangular inequality, the $L^{2}(\mu)$-norm of the difference is obviously bounded by

$$
\begin{aligned}
n^{-1 / 2} \sum_{m=0}^{n-1}\left\|e^{<\cdot, P_{t} \tau_{n, m} \xi>}\right\|_{2}\left|1-\exp \left(-\frac{1}{2 n} \int_{0}^{t} \xi\left(\frac{m+s}{n}\right)^{2} d s\right)\right| \\
\leq n^{-1 / 2} \sum_{m=0}^{n-1} e^{|\xi|_{2}^{2}} O_{\xi, t}\left(n^{-1}\right) \\
=O_{\xi, t}\left(n^{-1 / 2}\right),
\end{aligned}
$$


where we used Taylor's theorem, and the estimation

$$
\begin{aligned}
\left\|e^{\left.<\cdot, P_{\mathrm{t}} \tau_{n, m} \xi\right\rangle}\right\|_{2} & =e^{\left|P_{\mathrm{t}} \tau_{n, m} \xi\right|_{2}^{2}} \\
& \leq e^{\left|\tau_{n, m} \xi\right|_{2}^{2}} \\
& =e^{|\xi|_{2}^{2}}
\end{aligned}
$$

(ii) Now we prove that the $L^{2}(\mu)$-limit of $(5.5)$ is equal to the $L^{2}(\mu)$-limit of

$$
n^{-1 / 2} \sum_{m=0}^{n-1}<\cdot, P_{t} \tau_{n, m} \xi>
$$

By Taylor's theorem the difference of both terms is equal to

$$
n^{-1 / 2} \sum_{m=0}^{n-1}\left(\int_{0}^{1}(1-\lambda) e^{\lambda<\cdot, P_{t} \tau_{n, m} \xi>} d \lambda\right)<\cdot, P_{t} \tau_{n, m} \xi>^{2}
$$

The $L^{2}(\mu)$-norm of the last expression is bounded from above by

$$
n^{-1 / 2} \sum_{m=0}^{n-1} \int_{0}^{1}(1-\lambda)\left\|e^{\lambda<\cdot, P_{t} \tau_{n, m} \xi>}<\cdot, P_{t} \tau_{n, m} \xi>^{2}\right\|_{2} d \lambda \leq C_{1} t n^{-1 / 2}|\xi|_{\infty}^{2} e^{C_{2}|\xi|_{2}^{2}}
$$

where $C_{1}, C_{2}>0$ are constants, and we made use of Hölder's inequality and the estimates $\left|P_{t} \tau_{n, m} \xi\right|_{2} \leq t^{1 / 2} n^{-1 / 2}|\xi|_{\infty},\left|P_{t} \tau_{n, m} \xi\right|_{2} \leq|\xi|_{2}$.

(iii) Finally, by Lemma $5.2 n^{-1 / 2} \sum_{m=0}^{n-1} P_{t} \tau_{n, m} \xi$ converges in $L^{2}(\mathbb{R})$ to $\int_{0}^{1} \xi(u) d u \cdot 1_{[0, t]}$, which entails immediately that (5.6) converges in $L^{2}(\mu)$ to $B(t) \cdot \int_{0}^{1} \xi(u) d u$, as $n$ tends to infinity.

By hypothesis $1_{\{X \geq x\}}|\nabla X|_{2}^{-2} F(X)$ belongs to $L^{2}(\mu)$, and we have thus proved that the first of the two terms on the right hand side of (5.3) converges for every $\xi \in \mathcal{S}(\mathbb{R})$ to

$$
\left(\int_{\{X \geq x\}}|\nabla X|_{2}^{-2} F(X) B(t) d \mu\right) \cdot \int_{0}^{1} \xi(u) d u
$$

as $n$ tends to infinity.

Next we show that the second term on the right hand side of (5.3) converges to

$$
\left(\int_{\{X \geq x\}}|\nabla X|_{2}^{-2} D_{1_{[0, t]}} X d \mu\right) \cdot \int_{0}^{1} \xi(u) d u .
$$

To this end it is sufficient to prove that

$$
n^{-1 / 2} \sum_{m=0}^{n-1} D_{P_{t} \tau_{n, m} \xi} X: e^{\left\langle\cdot, P_{t} \tau_{n, m} \xi\right\rangle}:
$$


converges in $L^{2}(\mu)$ to $D_{\bar{\xi}_{10, t)}} X$, where we have set $\bar{\xi}:=\int_{0}^{1} \xi(u) d u$. We use the fact that for $h \in L^{2}(\mathbb{R}) X \in \mathcal{D}^{2,1}$, the mapping $h \longmapsto D_{h} X$ is linear, and the triangular inequality to estimate as follows

$$
\begin{aligned}
& \left\|n^{-1 / 2} \sum_{m=0}^{n-1} D_{P_{\mathrm{t}} \tau_{n, m} \xi} X: e^{\left\langle\cdot, P_{\mathrm{t}} \tau_{n, m} \xi\right\rangle}:-D_{\bar{\xi}_{1}[0, t]} X\right\|_{2} \\
& \quad \leq\left\|D_{\xi_{n, t}} X-D_{\bar{\xi}_{10, t]}} X\right\|_{2}+\left\|n^{-1 / 2} \sum_{m=0}^{n-1}\left(D_{P_{\mathrm{t}} \tau_{n, m} \xi} X\right)\left(: e^{\left.<\cdot, P_{\mathrm{t}} \tau_{n, m} \xi\right\rangle}:-1\right)\right\|_{2}
\end{aligned}
$$

where we have put $\xi_{n, t}:=n^{-1 / 2} \sum_{m=0}^{n-1} P_{t} \tau_{n, m} \xi$. Again by linearity, the first term on the right hand side is equal to

$$
\left\|D_{\xi_{n, t}-\bar{\xi} 1_{[0, t]}} X\right\|_{2} \text {. }
$$

It is clear (see above) that we can bound this from above by

$$
\left|\xi_{n, t}-\bar{\xi} 1_{[0, t]}\right|_{2}\|X\|_{2,1} \text {. }
$$

Now we can apply Lemma 5.2 which shows that this terms vanishes in the limit $n \longrightarrow+\infty$. Using Hölder's inequality we can bound the second term above by

$$
\begin{aligned}
n^{-1 / 2} \sum_{m=0}^{n-1}\left\|D_{P_{\mathrm{t}} \tau_{n, m} \xi} X\right\|_{4}\left\|: e^{<\cdot, P_{\mathrm{t}} \tau_{n, m} \xi>}:-1\right\|_{4} \\
\quad \leq \text { const. }\|X\|_{4,1} n^{-1 / 2} \sum_{m=0}^{n-1}\left|P_{t} \tau_{n, m} \xi\right|_{2}\left\|: e^{\left.<\cdot, P_{\mathrm{t}} \tau_{n, m} \xi\right\rangle}:-1\right\|_{4},
\end{aligned}
$$

where we applied Meyer's inequality (cf. Section 2) in the second step. Thus we get a bound of the form

$$
\text { const. } t^{1 / 2}|\xi|_{\infty}\|X\|_{4,1} n^{-1} \sum_{m=0}^{n-1}\left\|: e^{\left\langle\cdot, P_{t} \tau_{n, m} \xi>\right.}:-1\right\|_{4}
$$

for this term. With the help of Taylor's theorem, Hölder's inequality and $\left|P_{t} \tau_{n, m} \xi\right|_{2} \leq$ $t^{1 / 2} n^{-1 / 2}|\xi|_{\infty}$, it is straightforward to show that the last $L^{4}(\mu)$-norm is bounded by $O_{\xi, t}\left(n^{-1 / 2}\right)$, and therefore this term, too, converges to zero as $n$ tends to infinity.

Altogether we have shown that the $\mathcal{S}$-transform of (5.1) converges pointwise to the $\mathcal{S}$-transform of $\sigma(t, x) \cdot B(1)$, where $\sigma(t, x)$ is given by (5.2). As in the proof of Theorem 4.3, it remains to show a uniform bound on the $\mathcal{S}$-transform of $(5.1)$ evaluated at $z \xi, z \in \mathbb{C}$. But if one uses (5.3) and the preceding estimations, this is completely analogous to the arguments at the end of the rpoof of Theorem 4.3. The details are left to the interested reader.

We conclude this paper with an illustration of Theorem 4.3 and Theorem 5.1 by applying these results to solutions of one-dimensional, non-degenerate Itô equations. 
Let $a>0$ and $b$ be functions in $C^{\infty}(\mathbb{R})$, with all derivatives being bounded. Furthermore, let $x_{0} \in \mathbb{R}$, and consider the $\operatorname{SDE}$ (in Itô sense)

$$
\begin{aligned}
d X(t) & =a(X(t)) d B(t)+b(X(t)) d t \\
X(0) & =x_{0} .
\end{aligned}
$$

Then it is well-known (e.g., [IW 81]) that (4.2) has a unique solution $X$ satisfying (H.1). Moreover, it is proved in [Wa 84, Theorem 2.1] that $X(t), t>0$, admits (H.2). Finally, the assumption that $a>0$ implies that also (H.3) holds true for $X(t), t>0$, cf., e.g., [Wa 84 , Theorem 2.7]. Consider now the versions $X_{n, m}(t), n \in \mathbb{N}, m=0,1 \ldots, n-1$, of $X(t)$. We remark in passing that $X_{n, m}$ solves (4.2) with $B$ replaced by $B_{n, m}$.

Theorem 5.3. Let $x \in \mathbb{R}, t>0$, and let $X(t)$ be the solution of (5.7). Then

$$
\lim _{n \rightarrow+\infty} n^{-1} \sum_{m=0}^{n-1} \delta_{x} \circ X_{n, m}=\mathbb{E}\left(\delta_{x} \circ X\right)
$$

and

$$
\lim _{n \rightarrow+\infty} n^{-1 / 2} \sum_{m=0}^{n-1}\left(\delta_{x} \circ X_{n, m}-\mathbb{E}\left(\delta_{x} \circ X\right)\right)=\sigma(x, t) B(1)
$$

as strong limits in $(\mathcal{S})^{*}$, where

$$
\mathbb{E}\left(\delta_{x} \circ X\right)=\int_{\{X \geq x\}}|\nabla X|_{2}^{-2} F(X) d \mu
$$

and

$$
\sigma(x, t)=\int_{\{X(t) \geq x\}}|\nabla X(t)|_{2}^{-2}\left(F(X(t)) B(t)-D_{1_{[0, t]}} X(t)\right) d \mu,
$$

with

$$
F(X(t))=N X(t)+2|\nabla X(t)|_{2}^{-2}\left(\nabla^{2} X(t),(\nabla X)^{\otimes 2}\right)_{L^{2}\left(\mathbb{R}^{2}\right)}
$$

\section{Appendix}

In this Appendix we collect some results related to unitary transformations on the underlying Hilbert space $L^{2}(\mathbb{R})$.

Assume that $\tau$ is a linear unitary transformation on $L^{2}(\mathbb{R})$, such that its restriction to $\mathcal{S}(\mathbb{R})$ is a homeomorphism of $\mathcal{S}(\mathbb{R})$. By duality, $\tau$ and $\tau^{-1}$ extend to $\mathcal{S}^{\prime}(\mathbb{R})$ (weakly continuously). 
Lemma A.1. Let $h \in L^{2}(\mathbb{R})$, and assume that $\varphi \in \mathcal{D}^{p, 1}, p>1$. Then

$$
\left(D_{h} \varphi\right) \circ \tau=D_{\tau^{-1} h \varphi \circ \tau}
$$

Proof. It is sufficient to consider an everywhere defined, differentiable function $\varphi$ on $\mathcal{S}^{\prime}(\mathbb{R})$. Then, for $\omega \in \mathcal{S}^{\prime}(\mathbb{R})$,

$$
\begin{aligned}
\left(D_{\tau^{-1} h}(\varphi \circ \tau)\right)(\omega) & =\lim _{\lambda \rightarrow 0} \lambda^{-1}\left(\varphi \circ \tau\left(\omega+\lambda \tau^{-1} h\right)-\varphi \circ \tau(\omega)\right) \\
& =\lim _{\lambda \rightarrow 0} \lambda^{-1}(\varphi(\tau \omega+\lambda h)-\varphi(\tau \omega)) \\
& =\left(\left(D_{h} \varphi\right) \circ \tau\right)(\omega) .
\end{aligned}
$$

\section{Lemma A.2.}

a) Let $\varphi \in \mathcal{D}^{p, 1}, p \geq 1$, then

$$
|\nabla \varphi \circ \tau|_{2}=|\nabla \varphi|_{2} \circ \tau
$$

b) Let $\varphi \in \mathcal{D}^{p, 2}, p \geq 2$, then

$$
N(\varphi \circ \tau)=(N \varphi) \circ \tau
$$

and

$$
\left(\nabla^{2}(\varphi \circ \tau),(\nabla(\varphi \circ \tau))^{\otimes 2}\right)_{L^{2}\left(\mathbb{R}^{2}\right)}=\left(\nabla^{2} \varphi,(\nabla \varphi)^{\otimes 2}\right)_{L^{2}\left(\mathbb{R}^{2}\right)} \circ \tau
$$

Proof. We only give a proof of statement a), the proofs of the statements in b) are shown in a similar way. Let $\left\{e_{k}, k \in \mathbb{N}\right\}$ be a CONS of $L^{2}(\mathbb{R})$. Then

$$
\begin{aligned}
|\nabla(\varphi \circ \tau)|_{2}^{2} & =\sum_{k=1}^{\infty}\left|D_{e_{k}}(\varphi \circ \tau)\right|^{2} \\
& =\sum_{k=1}^{\infty}\left|D_{\tau e_{k}} \varphi\right|^{2} \circ \tau
\end{aligned}
$$

where we used Lemma A.1. But now $\left\{\tau e_{k}, k \in \mathbb{N}\right\}$ is another CONS of $L^{2}(\mathbb{R})$, and therefore the last expression equals $|\nabla \varphi|_{2}^{2} \circ \tau$. 


\section{References}

[Am 92] Amine, S.: Law of large numbers and central limit theorem for distributions on the Wiener space; Preprint (1992)

[HK 92] Hida, T., Kuo, H.-H., Potthoff, J. and Streit, L.: White Noise: An Infinite Dimensional Calculus. Manuscript for a monograph, to appear

[IK 81] Ikeda, N. and Watanabe, S.: Stochastic Differential Equations and Diffusion Processes. Amsterdam: North-Holland, Tokyo: Kodansha (1981)

[Ma 78] Malliavin, P.: Stochastic calculus of variation and hypoelliptic operators; in Proc. Int. Symp. on Stochastic Differential Equations; K. Itô (ed.). Tokyo: Kinokuniya, New York: Wiley (1978)

[Me 83] Meyer, P.A.: Quelques résultats analytiques sur le semigroupe d'OrnsteinUhlenbeck en dimension infinie; in Theory and Application of Random Fields; G. Kallianpur (ed.). Berlin, Heidelberg, New York: Springer (1983)

[PS 91] Potthoff, J. and Streit, L.: A characterization of Hida distributions; J. Funct. Anal. 101 (1991) 212-229

[PS 92] Potthoff, J. and Sundar, P.: Law of large numbers and central limit theorem for Donsker's delta function; Preprint (1992), to appear in Stochastics and Stochastics Reports

[Su 85] Sugita, H.: Sobolev spaces of Wiener functionals and Malliavin's calculus; J. Math. Kyoto Univ. 25 (1985) 31-48

[Wa 83] Watanabe, S.: Malliavin's calculus in terms of generalized Wiener functionals; in Theory and Application of Random Fields; G. Kallianpur (ed.). Berlin, Heidelberg, New York: Springer (1983)

[Wa 84] Watanabe, S.: Stochastic Differential Equations and Malliavin Calculus. Bombay: Tata Inst. of Fundamental Research (1984) 